\title{
A Pitfall in the Diagnosis of Unresectable Liver Metastases: Multiple Bile Duct Hamartomas (von Meyenburg Complexes)
}

\author{
David Fuks $^{\mathrm{a}}$ Jean-Philippe Le Mouel ${ }^{\mathrm{a}}$ Denis Chatelain ${ }^{\mathrm{b}}$ \\ Charles Sabbagh $^{\mathrm{a}}$ Fabien Demuynck $^{\mathrm{c}}$ Marie Brevet ${ }^{\mathrm{b}}$ \\ Olivier Brehant ${ }^{a}$ Eric Nguyen-Khac ${ }^{d}$ Thierry Yzet ${ }^{c}$ \\ Frederic Dumont $^{\mathrm{a}} \quad$ Pierre Verhaeghe $^{\mathrm{a}}$ \\ Jean-Marc Regimbeau ${ }^{a}$ \\ Departments of a Digestive Surgery, ${ }^{b}$ Pathology, 'Radiology and \\ ${ }^{\mathrm{d}}$ Gastroenterology, Amiens North Hospital, University of Picardy, Amiens, France
}

\section{Key Words}

Unresectable liver metastases · Multiple bile duct hamartomas · Von Meyenburg complexes

\begin{abstract}
Von Meyenburg complexes (VMC) are a cluster of benign liver malformations including biliary cystic lesions, with congenital fibrocollagenous stroma. This rare entity can mimick multiple secondary hepatic lesions. We report a case of a 56-year-old woman who had multiples liver lesions 12 years after operation for breast cancer. Biopsy of the hepatic lesion confirmed the diagnosis of VMC. Preoperative discovery of multiple gray-white nodular lesions scattered on the surface of the liver should not always contraindicate curative liver resection. The diagnosis of VMC should be known and confirmed with liver biopsy.
\end{abstract}

\section{Case Report}

A 56-year-old woman was operated in 1996 for breast cancer. Ten years later, levels of serum tumor marker CA15-3 progressively increased until $2 \mathrm{~N}$ although other serum tumor markers ( $\alpha$-fetoprotein, carcinoembryonic antigen, carbohydrate antigen 19-9) were normal. Axial contrast-enhanced multislice CT scan showed a solitary and hypovascular $4-\mathrm{cm}$ lesion located in the right lobe of the liver (segment VII). Liver MRI found this lesion in segment VII to be associated with multiple and small lesions with high signal intensity scattered throughout the liver especially in the subcapsular area in T2-weighted images (fig. 1a, b). After contrast injection, the multiple masses showed irregular progressive enhancement from arterial phase through delayed phase. Gastroscopy and coloscopy did 
not find new tumors and PET scan showed hyperfixation only on the hepatic lesion in segment VII. The diagnosis of a single liver metastasis of breast cancer was suspected although its characteristics were unusual (hypovascular lesion). The diagnosis of von Meyenburg complexes (VMC) was also suspected preoperatively, but the presence of multiple secondary hepatic lesions could not be dismissed because of breast cancer history. Percutaneous biopsy of the largest hepatic lesion found fibrosis but no malignant infiltration and could neither confirm the diagnosis of metastasis of breast cancer nor the diagnosis of VMC. Since this tumor had unusual characteristics, we performed laparoscopic assessment to obtain histological proof of malignancy. During laparoscopy, multiple gray-white nodular lesions (about 0.5$1 \mathrm{~cm}$ in diameter) were found on the surface of the liver (fig. 1c). A liver biopsy was performed because we feared multiple liver metastases (contraindication of curative resection). Histological examination confirmed the diagnosis of multiple benign VMC (biliary hamartomas) encompassed by major fibrous stroma. After conversion to laparotomy, right hepatectomy was performed and the pathological findings of the specimen confirmed the liver metastasis of the breast cancer in segment VII (without argument for cholangiocarcinoma) associated with multifocal, irregularly dilated tinged bile ducts. The patient was discharged from the hospital 9 days after surgery without complications.

\section{Discussion}

VMC is a cluster of benign liver malformations including biliary cystic lesions, with congenital fibrocollagenous stroma [1]. This entity corresponds to ductal plate malformation, which explains why they can be found in association with other congenital intrahepatic abnormalities, such as congenital hepatic fibrosis, Caroli's syndrome and polycystic liver disease. VMC is detected incidentally in 0.6 to $5.6 \%$ of reported autopsies [2]. VMC may be associated with an increased incidence of cholangiocarcinoma and cystic disease [3]. VMC are usually asymptomatic but clinical findings including jaundice, cholangitis, epigastralgias and fever have been reported [1,4]. Biliary hamartomas are known as either solitary or multiple millimetric lesions [3]. When these lesions are multiple, deep and larg (many millimeters in diameter), they can simulate secondary metastatic lesions particularly in case of cancer history and so contraindicate curative resection. Preoperative characteristics of VMC should be known. Ultrasound can show hypoechoic (larger cysts) and/or hyperechoic structures (smaller cysts) with 'comet-tail' echoes [5]. In some case, these hyperechoic structures correspond to small stones inside the dilated bile ducts. Nonenhanced CT shows hypodense, small hepatic nodules, scattered throughout both liver lobes, measuring less than $15 \mathrm{~mm}$ in diameter. The enhancement is variable on post-contrast CT images [6]. The best imaging to diagnose VMC is MRI with typically hypointense and well-defined lesions in T1-weighted images and hyperintense lesions in T2-weighted images $[3,6,7]$. Semelka et al. reported a thin rim enhancement on early postgadolinium images that persisted until late postgadolinium images [3]. VMC was suspected preoperatively in our patient, but confirmation of the diagnosis required further investigations particularly because of cancer history. Macroscopically, VMC are grayish white nodules measuring 1 to $15 \mathrm{~mm}$ in size that are scattered throughout the liver, particularly below the capsule of the liver, in the periportal region of hepatic lobules. A definitive diagnosis of VMC requires histopathologic examination of the specimen because its findings are nonspecific and easily confused with malignant liver diseases [4]. Microscopically, VMC are characterized by cystic dilatations of the bile duct or clusters of mature bile duct of various sizes, periductal glands and encompassed by fibrous stroma [8].

Preoperative discovery of multiple gray-white nodular lesions scattered on the surface of the liver should not always contraindicate curative liver resection. The diagnosis of VMC should be known and confirmed by liver biopsy. 


\begin{tabular}{r|l|l|l} 
Case Reports $/$ h & $\begin{array}{l}\text { Case Rep Gastroenterol 2009;3:198-201 } \\
\text { D01: 10.1159/000220819 }\end{array}$ & Published online: July 31, 2009 & $\begin{array}{l}\text { O 2009 S. Karger AG, Basel } \\
\text { ISSN 1662-0631 } \\
\text { www.karger.com/crg }\end{array}$ \\
\hline
\end{tabular}

Fig. 1. a Axial T2-weighted liver MRI showing numerous and small lesions with high signal intensity scattered throughout the liver especially in the subcapsular area of the right liver lobe. b Coronal MR cholangiopancreatography demonstrating no communication between the cystic lesions and the normal biliary system. c Specimen of right hepatectomy showing gray-white nodular lesions (about $0.5 \mathrm{~cm}$ in diameter) scattered on the surface of the liver. $\mathbf{d}$ Specimen of right hepatectomy showing gray-white nodular lesions throughout the liver parenchyma associated to a large lesion (liver metastasis) with retraction of the liver capsule. e Microscopic view showing multiple bile ducts with slightly dilated lumens embedded in the collagenous stroma (hematoxylin and eosin, $\times 100$ ).
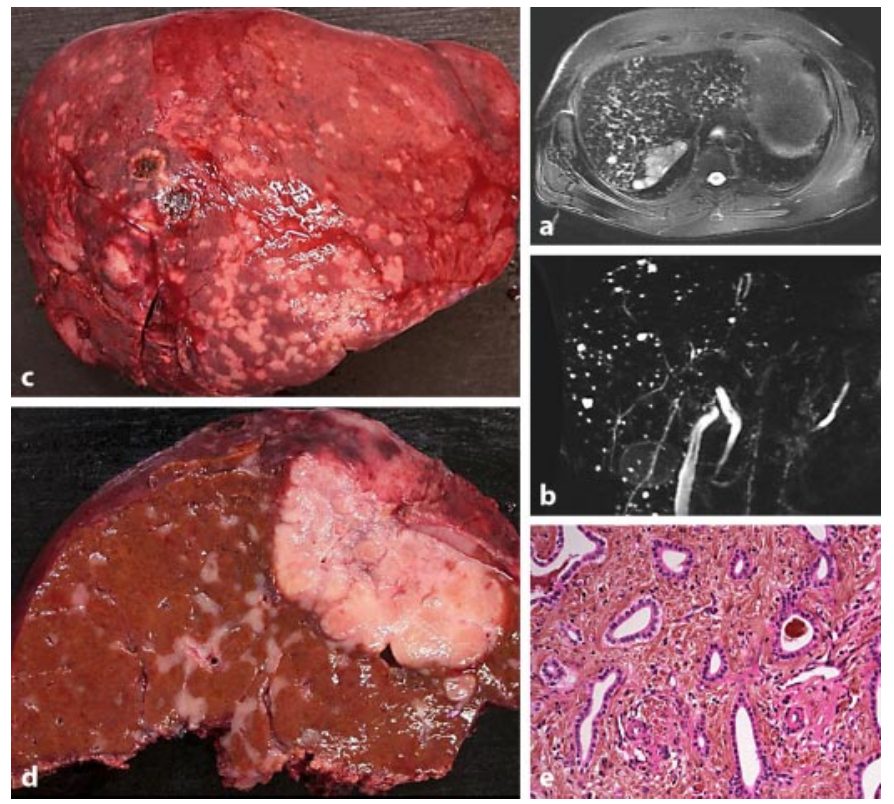


\section{References}

1 Karahan OI, Kahriman G, Soyuer I, Ok E: Hepatic von Meyenburg complex simulating biliary cystadenocarcinoma. Clin Imaging 2007;31:50-53.

2 Redston MS, Wanless IR: The hepatic von Meyenburg complex: prevalence and association with hepatic and renal cysts among 2,843 autopsies. Mod Pathol 1996;9:233-237.

-3 Semelka RC, Hussain SM, Marcos HB, Woosley JT: Biliary hamartomas: solitary and multiple lesions shown on current MR techniques including gadolinium enhancement. J Magn Reson Imaging 1999;10:196-201.

-4 Aoki S, Okayama Y, Kitajima Y, Hayashi K, Imai H, Okamoto T, Akita S, Gotoh $\mathrm{K}$, Ohara H, Nomura T, Joh T, Yokoyama Y, Itoh M: Intrahepatic biliary papilloma morphologically similar to biliary cystadenoma. J Gastroenterol Hepatol 2005;20:321-324.

5 Tröltzsch M, Borte G, Kahn T, Mössner J, Teich N: Non-invasive diagnosis of von Meyenburg complexes. J Hepatol 2003;39:129.

-6 Mortelé B, Mortelé K, Seynaeve P, Vandevelde D, Kunnen M, Ros PR: Hepatic bile duct hamartomas (von Meyenburg complexes): MR and MR cholangiography findings. J Comput Assist Tomogr 2002;26:438-443.

7 Cheung YC, Tan CF, Wan YL, Lui KW, Tsai CC: MRI of multiple biliary hamartomas. Br J Radiol 1997;70:527-529.

-8 Zen Y, Terahata S, Miyayama S, Mitsui T, Takehara A, Miura S, Nobata K, Kitao A, Kakuda K, Kiyohara K, Nakanuma Y: Multicystic biliary hamartoma: a hitherto undescribed lesion. Hum Pathol 2006;37:339-344. 